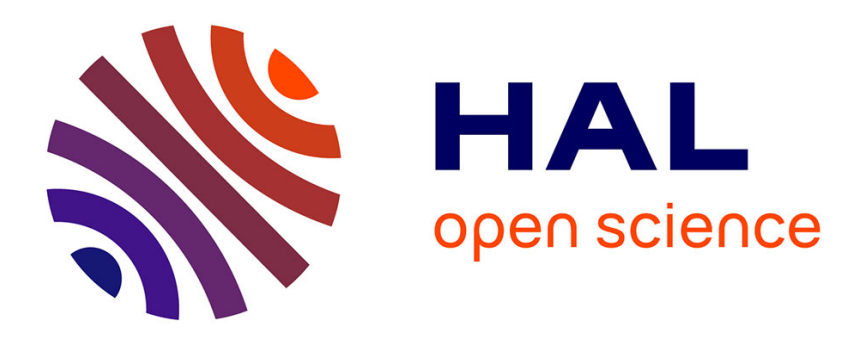

\title{
On the Stochastic Properties of Carbon Futures Prices
} Julien Chevallier, Benoît Sévi

\section{To cite this version:}

Julien Chevallier, Benoît Sévi. On the Stochastic Properties of Carbon Futures Prices. 2012. halshs00720166v2

\section{HAL Id: halshs-00720166 \\ https://shs.hal.science/halshs-00720166v2}

Preprint submitted on 21 Oct 2012

HAL is a multi-disciplinary open access archive for the deposit and dissemination of scientific research documents, whether they are published or not. The documents may come from teaching and research institutions in France or abroad, or from public or private research centers.
L'archive ouverte pluridisciplinaire HAL, est destinée au dépôt et à la diffusion de documents scientifiques de niveau recherche, publiés ou non, émanant des établissements d'enseignement et de recherche français ou étrangers, des laboratoires publics ou privés. 


\title{
On the Stochastic Properties of Carbon Futures Prices* \\ Julien Chevallier ${ }^{\dagger}$ \\ Université Paris 8 \\ Benoît Sévi ${ }^{\ddagger}$ \\ Aix-Marseille School of Economics
}

Revised Version: October 2012

\begin{abstract}
Pricing carbon is a central concern in environmental economics, due to the importance of emissions trading schemes worldwide to regulate pollution. This paper documents the presence of small and large jumps in the stochastic process of the $\mathrm{CO}_{2}$ futures price. The large jumps have a discrete origin, i.e. they can arise from various demand factors or institutional decisions on the tradable permits market. Contrary to the previously established literature, we show that the stochastic process of the carbon futures prices does not contain a continuous component (Brownian motion). The results are derived by using high-frequency data in the activity signature function framework (Todorov and Tauchen $(2010,2011)$ ). The implication is that the carbon futures price should be rather modelled as an appropriately sampled, centered Lévy or Poisson process. The pure-jump behavior of the carbon price could be explained by the lower volume of trades on this allowance market (compared to other highly liquid financial markets).
\end{abstract}

JEL Classification: C14; C32; G1; Q4

Keywords: Carbon Price; Stochastic Modeling; Activity Signature Function.

\footnotetext{
*Acknowledgements: Helpful comments were received from seminar participants at the 10th Envecon Applied Environmental Economics Conference in London (UKNEE). We wish to thank ECX for providing the data. The usual disclaimer applies.

$\dagger$ Corresponding Author. Université Paris 8, LED. 2 rue de la Liberté, 93526 Saint-Denis Cedex, France. Tel: +33(0)149407386. Fax: +33(0)149407255. julien.chevallier04@univ-paris8.fr

¥Aix-Marseille University (Aix-Marseille School of Economics), CNRS \& EHESS. Château La Farge, Route des Milles, 13290 Aix-en-Provence Les Milles, France. Email: benoit.sevi@univ-amu.fr
} 


\section{Introduction}

The theory behind tradable permits markets certainly found its roots in the writing of environmental economists (Dales (1968), Montgomery (1972)). Dynamic properties were then progressively established in the very same literature (Cronshaw and Kruse (1996), Ellerman (2005), Kling and Rubin (1997), Leiby and Rubin (2001), Maeda (2004), Rubin (1996), Schennach (2000), Stevens and Rose (2002)).

Recently, a parallel mathematical literature has developed to establish the properties of tradable permits programs, in the wider context of the European Union Emissions Trading Scheme (EU ETS) and the price formation of European Union Allowances (EUAs). These papers are typically interested in recovering the stochastic properties of allowance prices, either to model the dynamic price equilibrium (Carmona et al. (2009, 2010)), or to address the question of derivatives valuation in emissions markets (Chesney and Taschini (2012), Çetin and Verschuere (2009), Borovkov et al. (2011), Carmona and Hinz (2011), Hinz and Novikov (2010)).

With the notable exception of Seifert et al. (2008), there seems to be a gap in the environmental economics literature to document these stochastic properties of carbon prices. Some papers have contributed to the econometric modeling of carbon assets in the ARCH framework (Paolella and Taschini (2008), Benz and Trück (2009)), but only Daskalakis et al. (2009) have derived some stochastic properties.

Reasons for stochastic modeling of carbon prices are amongst other expected allowance demand and fluctuating fuel prices, which potentially lead to a change in the merit order of power production, fuel-switch costs, weather changes, economic growth, etc. (Seifert et al. (2008), Carmona et al. (2009)). The Brownian motion assumption is intended to capture these uncertainties in a simplified way. Other popular stochastic processes include mean-reversion (Vasicek, OrnsteinUhlenbeck) and/or jumps (Poisson, Lévy, Bernoulli).

For modeling emission allowances, it appears necessary to identify a stochastic process which can map the empirical properties of the price as a proxy for marginal abatement costs. Uncovering the stochastic properties of carbon futures prices is also a necessary step for building an equilibrium pricing model for futures, choosing the appropriate option pricing model, and evaluating investment decisions - for example through a real-option valuation approach (Daskalakis et al. (2009), Zhu et al. (2009)).

The goals pursued by this paper are twofold: (i) review the main results on the stochastic properties of carbon prices derived in the mathematical finance literature, and (ii) apply an original empirical method to establish the stochastic properties of carbon futures prices by means of the activity signature function (Todorov and Tauchen $(2010,2011)$ ).

Our empirical work relies on the Activity Signature Function (ASF) - a nonparametric methodology developed in Todorov and Tauchen (2010) - to investigate the underlying stochastic process for the price of carbon. In short, the methodology is able to deliver evidence about the presence of a Brownian motion and/or the presence of small, medium or large jumps with finite or infinite activity using intraday data on transaction prices in the financial futures market of emissions allowances 
for carbon dioxide. A number of alternatives to the suggested procedure have been developed in the statistical literature, which also rely on high-frequency data. In particular, Aït-Sahalia and Jacod (2010) study theoretically the issue of detecting the presence of a Brownian motion in the stochastic process. Jing et al. (2012) and Kong (2012) have extended the test in Ait-Sahalia and Jacod (2010) with a similar null hypothesis that a continuous component is present. ${ }^{1}$ The advantage of using the empirical approach in Todorov and Tauchen (2010) is simple, as their procedure includes the above-mentioned contributions and, in addition, provides some evidence about the nature of jumps, if any, in the stochastic process. Moreover, the authors propose a formal test to assess the presence of a continuous component that has good finite-sample properties. As such, the ASF methodology is more satisfactory than other existing approaches, thereby motivating our choice in the present paper.

In recent years, many pure jump or jump-diffusion models have been suggested in the economic and statistical literatures to deal with (possibly large) discontinuities in price processes; see the discussion in Todorov and Tauchen $(2010,2011)$ and the reference textbook by Cont and Tankov (2004). Adding a jump component to a continuous component (leading to a mixture model) or considering a jump component only allows to fit the data better than with a continuous component only. $^{2}$ In addition, given the presence of jumps in the data, pure jump models are preferred by users as they are easier to handle for practical applications such as derivatives pricing or real-life problems such as valuation of insurance contracts (see Ballotta (2005) or Kassberg et al. (2008)) or real-option valuation (Martzoukos and Trigeorgis, 2002). ${ }^{3}$ As a simple motivating example, let us consider European energy utilities that have to plan future investments. The utilities are interested in the modelling of carbon prices because different choices will lead to distinct conclusions. Indeed, considering a simple Brownian motion or one of the existing pure jump models (or alternatively a jump-diffusion) has a considerable impact on the valuation of projects even if these projects have a several-year horizon. ${ }^{4}$ In this context, Martzoukos and Trigeorgis (2002) provide methodologies in the case of real options to deal with the issue of rare events that may be represented by jumps in the stochastic process. Their results show the very significant impact of jumps, in particular for complex real options such as growth options or extension options. European utilities may also be interested in optimal hedging in the carbon market, where the hedging decision depends on the stochastic properties of the underlying security.

Our central contribution is to show that all stochastic processes for carbon futures prices that have been suggested so far in the literature, and which explicitly include a continuous component (Brownian motion), are not credible candidates in light of the empirical evidence analyzed in our paper. Based on our data sample, we show that there is no continuous component in the carbon

\footnotetext{
${ }^{1}$ Aït-Sahalia and Jacod (2010) also suggest an alternative test, where the null hypothesis is the absence of a continuous component. The difference between the tests lies in their theoretical and finite-sample properties.

${ }^{2}$ The large empirical evidence about financial data is discussed in Cont and Tankov (2004), among others.

${ }^{3}$ Other examples of pure jump or jump-diffusion models are provided in Jing et al. (2012). The authors emphasize that such models have applications far beyond the financial domain.

${ }^{4}$ The interested reader may look at the Section 2 in Jing et al. (2012), where distributions from several processes of different nature are plotted. The distributional aspect of pure-jump or diffusion is strikingly different, thereby motivating the investigation of the fine nature of the underlying process for financial as well as for non-financial (real-world) applications.
} 
futures price process.

Our findings are based on nonparametric estimates which use intraday data to draw the finest conclusion about the price process underlying the carbon price. Therefore, our approach contrasts with previous parametric studies that only calibrate their models by using at best daily data and, as such, disregard most of the available information.

Since 2005, the $\mathrm{CO}_{2}$ price constitutes one of the central pollution price references in environmental economics (with for instance $\mathrm{SO}_{2}$ ), which is being traded every day and worldwide. Our findings therefore have important implications for the modelling of such a price process.

The rest of the paper is organized as follows. Section 2 details the previous studies. Section 3 contains the empirical work. Section 4 concludes. An Appendix is included to detail the key characteristics of stochastic processes.

\section{Previous studies}

Several studies have attempted to identify the stochastic processes of carbon prices (both spot and futures). We briefly recall the main findings from this literature for continuous- and jumpdiffusion models. The interested reader may find an overview of the key characteristics of stochastic processes (with/without jumps) in the Appendix.

\subsection{Continuous-diffusion models}

\subsubsection{Spot price}

Seifert et al. (2008) first derived the characteristic partial differential equation that fully describes the solution of a theoretical emissions trading model for $\mathrm{CO}_{2}$ emissions certificates under the EU ETS. The goal of their pioneering analysis could be viewed as determining the expected value of an optimal policy given the total expected emissions, where the $\mathrm{CO}_{2}$ spot price is set equal to the companies' marginal abatement costs.

To address such standard problem in environmental economics, they resort to a controlled stochastic process $x_{t}$, which describes the expectation of the cumulative emissions over the whole trading period at time $t$, taking into account both known emissions and abatement actions in the past up to time $t$, as well as expected future emissions from $t$ onwards. The volatility of the uncontrolled stochastic part of the process is determined by a drift and the volatility of the underlying emissions rate.

Insofar as the modeling of the emissions rate is concerned, the study by Seifert et al. (2008) is compatible with at least three of the typical stochastic processes mentioned in the Appendix:

1. an arithmetic Brownian motion without drift,

2. a white noise process, and

3. an Ornstein-Uhlenbeck process. 
In a risk-neutral special case ${ }^{5}$, the analytically derived properties of the equilibrium $\mathrm{CO}_{2}$ spot price yield to the following results:

- the spot price may not rise above the penalty (because $\mathrm{CO}_{2}$-regulated companies would no longer increase abatement but would rather pay the cheaper penalty);

- the spot price never actually reaches zero (which reflects the option value of emissions certificates);

- companies are always willing to abate emissions in order to mitigate some of the expected penalty costs (resulting in a positive spot price).

These results can be further generalized to the case of banking behavior on the emissions market ${ }^{6}$. In terms of volatility dynamics, the authors are able to show that the volatility of the spot price:

- approaches zero when $x_{t}$ is far away from the initial endowment;

- increases with positive changes in the penalty cost, abatement costs, and emissions rate volatility;

- should be bounded between zero and the penalty.

Seifert et al. (2008) suggest to calibrate this $\mathrm{CO}_{2}$ spot price process to market data, as an input to pricing $\mathrm{CO}_{2}$ derivatives. A potential buyer or seller of an option contract on $\mathrm{CO}_{2}$ emission allowances usually will employ some underlying spot price process in order to value the contract. In case of misspecification of the volatility process, significant mispricing errors can occur. Besides, the spot price process can be used as an additional component in a power price model with emissions trading.

\subsubsection{Futures price}

Zhu et al. (2009) have proposed a single stochastic model to represent the underlying stochastic properties of carbon futures prices. Mean-reversion (e.g. Vasicek process) is used to capture the major stochastic features exhibited in carbon futures prices. Their model can be seen as representing the carbon process in the risk-neutral world. When futures prices and implied volatilities of vanilla options are available (and liquid enough), the market data can be used to calibrate the model $^{7}$.

Carmona and Hinz (2011) have developed another approach to model carbon futures prices. They consider that the futures price process follows the cumulative distributive function of the standard normal distribution, and write their stochastic differential equation with a Brownian motion process. By calibrating this model to historical daily prices of a futures contract written on $\mathrm{CO}_{2}$ futures with maturity in December 2012, and by applying it to the valuation of European

\footnotetext{
${ }^{5}$ Note that these results can be extended to the potential effect of risk-aversion on $\mathrm{CO}_{2}$ prices.

${ }^{6}$ Banking consists in saving certificates for future use, by emitting less or abating more than would be required to cover the emissions of the current year.

${ }^{7}$ The authors typically use Monte-Carlo simulations or trinomial tree methods to study or estimate costs or risks directly dependent on the futures carbon prices.
} 
call options, the authors demonstrate that their model is capable of matching the observed historical or implied volatility of the underlying allowance futures price. As such, it possesses desirable properties from a hedging perspective, as well as for efficient option valuation schemes.

With reference to the key properties of stochastic processes presented in the Appendix, it is particularly interesting to notice that both categories of continuous diffusion models:

- Brownian motions,

- Mean-reversion processes (Vasicek and Ornstein-Uhlenbeck),

have been experimented to describe carbon spot and futures prices in previous literature. In Section 3, we will discuss whether it is indeed appropriate to retain these characteristics, which are common to model commodities price behavior ${ }^{8}$, also in the context of carbon prices.

Next, we review papers modeling explicitly the occurrence of jumps.

\subsection{Jump-diffusion models}

Identifying jumps in a stochastic process is important because if it has implications for risk management, option pricing, portfolio selection and has consequences for optimal hedging strategies. Indeed, when computed using simulation techniques, the quantiles sensibly differ when draws are from a continuous, or continuous plus jump distribution. Similarly, portfolio selection can be dramatically modified when some assets in the investment universe are potentially jumping (Liu et al. (2003), Cvitanić et al. (2008)).

\subsubsection{Spot price}

The first theoretical pricing of emissions allowances spot prices with jumps is given by Dannenberg and Ehrenfeld (2011) under a discretized form of the standard Vasicek process. After a stochastic shock occurring between $t$ and $t+1$, the spot market price is 'pulled back' towards the mean level by the mean reversion formula. The mean reversion rate stands for the strength with which the price is drawn back to the mean. This modelling is therefore close in its spirit to Zhu et al. (2009), as seen in Section 2.1.2.

Jumps can affect the allowance price due to the arrival of new information, which fundamentally changes the expectation of the marginal abatement cost. The change of expectation can be quite abrupt in this case. To account for jumps, the mean of the process is shifted by this new information.

The jump variable $J$ acts like a switch: either a shift of the mean-reversion level occurs from $t$ to $t+1(J=1)$ or not $(J=0)$. $J$ follows a Bernoulli process, with $p$ the probability of a shift

\footnotetext{
${ }^{8}$ The rationale for mean-reversion is that the price of a commodity tends to be pulled back to its production cost plus a margin (Schwartz (1997), Schwartz and Smith (2000)). For commodities such as energy, the mean is determined by the marginal cost of production and the extent of demand. In the short-run, there can be deviations from this arithmetic mean, but in the long-run the price converges towards the marginal costs of production as a result of competition among the producers. That is why the modeling of energy prices using the mean-reversion process is quite common.
} 
causing information to emerge. If the mean-reversion level is shifted to a new state, this level is bounded above and below. For the modeling of the new mean-reversion level, a PERT distribution is chosen ${ }^{9}$. This modeling of the jump size is chosen to achieve an approximately unbiased price modelling. Overall, Dannenberg and Ehrenfeld (2011) suggest to adopt a Mean-Reversion Jump Diffusion (MRJD) process for allowance spot prices.

Çetin and Verschuere (2009) note that Seifert et al. (2008) avoid the natural complication of an equilibrium analysis by assuming every market participant risk-neutral or the existence of a representative agent with a logarithmic utility, thereby reducing the problem to a central planner who aims to maximize the total profit of all agents. By contrast, they avoid the equilibrium approach to spot prices, but use the no-arbitrage principle between the spot and forward prices of carbon allowances ${ }^{10}$.

Without jumps, the $\mathrm{CO}_{2}$ spot price is a continuous process satisfying a stochastic differential equation (SDE) with constant, drift and a standard Brownian motion.

Interestingly, the net position of the allowance market - i.e. the extent to which the market is globally long or short of allowances ${ }^{11}$ - is governed by a Markov chain ${ }^{12} \theta$ which takes values in $\{-1,1\}: \theta_{t}=1\left(\theta_{t}=-1\right)$ corresponds to the market being long (short) at time $t$. To sum up, the price process of carbon allowances depends on two sources of uncertainty:

- the Brownian motion, and

- the net position of the allowance market.

Jumps are introduced under the form of intermediate announcements regarding the market's net position. Suppose at some $t_{0}<T$ the true position of the allowance market is revealed. Typically, there will be a jump at time $t_{0}$, and the jump size is modelled after the Lévy's characterization of Brownian motion.

By numerical implementation ${ }^{13}$, the authors illustrate how intermediate announcements of a long position can cause a sharp decline in $\mathrm{CO}_{2}$ prices. This situation mimics the information release in April 2006, which revealed that National Allocation Plans were so generous that there was almost no possibility for the market to end up short at the end of the period (Ellerman and Buchner (2008)). Hence, the model is satisfactory in capturing the main features observed for carbon spot prices during the first phase (2005-2007) of the EU ETS without banking and borrowing.

Borovkov et al. (2011) also propose a model of allowance spot pricing in the presence of jumps. According to the current practice of the EU ETS, revised decision on the amount of allocated

\footnotetext{
${ }^{9}$ The PERT distribution is a version of the beta distribution. We leave to the interested reader the explanation of the PERT distribution in the original article.

${ }^{10}$ By assuming an exogenous price process for the forward contract.

${ }^{11}$ Every year, the European Commission aggregates submitted emissions data and compares this to the quantity of allowances surrendered. The processing of emissions data for the entire zone takes a couple of months, and announcements on the market's net position are not released until mid-April.

${ }^{12}$ This mathematical system is widely used in business cycle analysis to explain the transitions from one state of the economy to another (e.g. boom or bust), between a finite or countable number of possible states.

${ }^{13}$ The calculation of the carbon spot price can be calculated in a Monte Carlo routine.
} 
certificates may yield to a jump of allowance market prices. Similarly, a sudden change in demand for production goods and/or price for fuel can result in the pollution levels changing dramatically, which impacts the allowance price. The state variable process is dependent upon:

- a pre-specified abatement function,

- the volatility level of the carbon price,

- a Brownian motion adapted to a general jump-diffusion process, and

- an adapted random Poisson measure.

Their approach allows to model jumps with great flexibility, as the distribution of the jump is state- and time-dependent. The authors establish the existence of a unique solution for the stochastic differential equation. Then, by means of simulation, they conclude that in presence of jumps the allowance spot price tends to converge slower than expected to its boundary value. The main reason is that, following jumps, agents can revise their anticipations towards a sudden increase or decrease in pollution emissions (which impacts directly the allowance price). Similar modeling results can be found in Hinz and Novikov (2010).

\subsubsection{Futures price}

To capture the dynamics of $\mathrm{CO}_{2}$ prices, Daskalakis et al. (2009) investigate some descriptive statistics of $\mathrm{CO}_{2}$ price series, which shed doubts on the validity of the standard Brownian motion assumptions. That is why they also examine the ability of various popular jump-diffusion processes in capturing the dynamics of $\mathrm{CO}_{2}$ prices. Their analysis starts first with the investigation of the dynamics of the carbon spot price, which is linked to futures through an inter-Phase trading equation ${ }^{14}$.

The drift, the diffusion and the jump coefficients are assumed to be general functions of time and the $\mathrm{CO}_{2}$ spot price. The jump component is controlled by a Poisson process with constant arrival parameter.

After conducting a 'horse race' between various configurations ${ }^{15}$, the authors find that the carbon spot price is better approximated by a Geometric Brownian motion process augmented by Jumps (GBMPJ).

Their results clearly indicate that the GBMPJ model performs better against competing models. The addition of jumps improves significantly the performance of most models, since all jumpdiffusion processes outperform their diffusion counterparts. Overall, their findings indicate that $\mathrm{CO}_{2}$ spot prices have a proportional, non-mean reverting structure with jumps, i.e., they are subject to large movements that cannot be explained by standard diffusion processes ${ }^{16}$.

Besides, it is worth noting that the addition of mean-reversion appears to decrease the goodnessof-fit, especially in the case of jump-diffusion models. This finding stands therefore in sharp contrast

\footnotetext{
${ }^{14}$ This setting can be seen as tailored to the constraints of the EU ETS during 2005-2007, when the inter-Phase I and II intertemporal transfer of allowances had been banned.

${ }^{15}$ Namely, Geometric Brownian motion process, Mean-reverting square-root process, Mean-reverting logarithmic process, Constant-elasticity of variance, Geometric Brownian motion process augmented by Jumps, and Meanreverting square-root process augmented by Jumps.

${ }^{16}$ Note, however, that the authors do not investiagte the presence of infinite activity jumps (as in our setting).
} 
to the approaches developed by Dannenberg and Ehrenfeld (2011), Lin and Lin (2007) and Zhu et al. $(2009)^{17}$.

Then, Daskalakis et al. (2009) derive a pricing formula for inter-Phase emission allowance futures ${ }^{18}$. Assuming a non-zero mean-reverting convenience yield, the inter-Phase carbon futures price is finally shown to be dependent upon the current level of convenience yield, the price of a zero-coupon bond with maturity at time $T$, the speed of mean-reversion of the convenience yield, the long-run mean yield, the convenience yield market price of risk, and the variance of the change in the marginal convenience yield. According to the authors, such a two-factor model that assumes a jump diffusion process for the underlying and a stochastic, mean-reverting convenience yield provides a satisfactory formula for the pricing of inter-Phase carbon futures.

Again, with reference to our presentation of stochastic processes in the Appendix, we have been successful in identifying the following classes of jump diffusion models:

- Poisson process,

- Lévy process,

- Bernoulli process,

as potential candidates for the stochastic properties of carbon futures prices according to previous literature.

\subsection{Summary}

Table 1 provides a useful summary of the findings from previous studies:

Table 1: Stochastic properties of carbon prices in previous literature

\begin{tabular}{|c|c|c|c|c|c|c|}
\hline Diffusion & \multicolumn{3}{|c|}{ Continuous } & \multicolumn{3}{|c|}{ Jump } \\
\hline Process & $\begin{array}{c}\text { Brownian } \\
\text { motion }\end{array}$ & Vasicek & $\begin{array}{l}\text { Ornstein- } \\
\text { Uhlenbeck }\end{array}$ & Poisson & Lévy & Bernoulli \\
\hline Seifert et al. (2008) & $\mathrm{X}$ & & $\mathrm{X}$ & & & \\
\hline Zhu et al. (2009) & $\mathrm{X}$ & $\mathrm{X}$ & & & & \\
\hline Carmona and Hinz (2011) & $\mathrm{X}$ & & & & & \\
\hline Çetin and Verschuere (2009) & $\mathrm{X}$ & & & & $\mathrm{X}$ & \\
\hline Borovkov et al. (2011) & $\mathrm{X}$ & & & $\mathrm{X}$ & & \\
\hline Hinz and Novikov (2010) & $\mathrm{X}$ & & & $\mathrm{X}$ & & \\
\hline Carmona et al. (2009) & $\mathrm{X}$ & & $\mathrm{X}$ & & & \\
\hline Daskalakis et al. (2009) & $\mathrm{X}$ & & & $\mathrm{X}$ & & \\
\hline Lin and Lin (2007) & $\mathrm{X}$ & $\mathrm{X}$ & & $\mathrm{X}$ & & \\
\hline Dannenberg and Ehrenfeld (2011) & $\mathrm{X}$ & $\mathrm{X}$ & & & & $\mathrm{X}$ \\
\hline
\end{tabular}

As discussed in Section 2.1.2 and Section 2.2.2, controversy remains on the usefulness of modeling the carbon price process with mean-reversion. Besides, the inclusion of jumps (either under

\footnotetext{
${ }^{17}$ While the modeling results of Dannenberg and Ehrenfeld (2011) and Zhu et al. (2009) have been covered, we briefly mention here the findings of Lin and Lin (2007). They model carbon dioxide spot prices as a result of mean-reversion with varying trends, combined with state-dependent price jumps and volatility structure. Globally, their results show that mean-reversion with state-dependent price jumps performs the best in forecasting the $\mathrm{CO}_{2}$ futures prices.

${ }^{18}$ I.e. with banking. Results with no banking are also provided.
} 
the form of Poisson- or Lévy-like processes) appears as a salient characteristic of most models developed.

At this stage, it is striking to remark that all models so far (even those including jumps) have used the Geometric Brownian motion assumption. This preliminary remark will be very important later in the empirical section regarding the main conclusion of our paper.

Next, we develop our own empirical investigation of the stochastic properties of carbon futures prices through the use of high-frequency data.

\section{$3 \quad$ Empirical evidence}

The rest of the paper is focused on determining the stochastic properties of carbon futures prices by using the activity signature function methodology. We first expose briefly the methodology, and then present the data and our empirical findings along with a sensitivity analysis of our results to the modeling choice. Finally, a discussion on the implications of our work in environmental economics is developed.

\subsection{Methodology}

Todorov and Tauchen (2010) define a new concept termed Activity Signature Function that is constructed from discrete observations of a continuous-time process. The ASF estimates the activity of the underlying stochastic process, and helps in selecting an appropriate process for the time series of interest. The main idea behind the ASF is to use the generalization by Ait-Sahalia and Jacod (2009b) of the Blumenthal-Getoor (1961) index which allows to 'classify the different processes used in continuous-time modeling in the following order from low to high activity: finite activity jumps, finite variation (but infinite activity) jumps, absolutely continuous processes, jumps of infinite variation and continuous martingales.' (Todorov and Tauchen (2010), p. 125). Another property of interest is that the most active component will dictate the value of the index. Todorov and Tauchen (2010) give the following example: if the activity index takes a value of 2 , the process contains a continuous martingale, while otherwise it is of a pure-jump type. We do not detail here why a value of 2 corresponds to a continuous martingale, and refer instead the interested reader to the original contributions of Aït-Sahalia and Jacod (2009b) and papers cited therein.

The models suggested in the literature differ in whether the stochastic process contains continuous martingale and/or jumps. Hence, the general form is as follows:

$$
\begin{aligned}
Y_{t} & =\int_{0}^{t} b_{1 s} d s+\int_{0}^{t} \sigma_{1 s} d W_{s} \\
X_{t} & =\int_{0}^{t} b_{2 s} d s+\int_{0}^{t} \int_{\mathbb{R}} \sigma_{2 s-} \kappa(\chi) \tilde{\mu}(d s, d x)+\int_{0}^{t} \int_{\mathbb{R}} \sigma_{2 s-} \kappa^{\prime}(\chi) \mu(d s, d x) \\
Z_{t} & =X_{t}+Y_{t}
\end{aligned}
$$


with $\int_{0}^{t} b_{1 s} d s$ and $\int_{0}^{t} b_{2 s} d s$ the drift terms in respectively $Y$ and $X, W_{t}$ a standard Brownian motion, $\mu$ a jump measure, $\kappa(\chi)$ a continuous truncation function. Stochastic volatility is generated through a time-varying jump size $\sigma_{2 t}$ and the time-varying intensity of the jumps. In Eq.(1), the first, second and third lines represent - respectively - a continuous model, a pure-jump model, and a continuous plus jumps model (mixture model).

As mentioned in the Introduction, the idea to measure the activity of the process via the activity signature function is to compute the realized power variation at two different frequencies. Asymptotic results in Aitt-Sahalia and Jacod (2009b) show that the rate of convergence for realized power variation, as we sample more frequently, only depends on the activity of the stochastic process. As a consequence, 'the ratio of the realized power variations computed over different scales, and hence the activity signature function, identifies the activity of the observed process'. (Todorov and Tauchen (2010), p. 126). Formally, let $x_{t, i}, i=1,2, \ldots,\left[1 / \Delta_{n}\right]$, denote the $i$ th increment of the process over the period $t$ (with financial data, the increment is simply a return that is computed as the difference of the logarithms of prices). The increments are considered at regularly spaced times with a sampling interval $\Delta_{n}$ so we have $\left[1 / \Delta_{n}\right]$ for the period $t$. The realized power variation at power $p$ is defined as:

$$
V\left(p, \Delta_{n}\right)=\sum_{i=1}^{\left[1 / \Delta_{n}\right]}\left|x_{t, i}\right|^{p}
$$

The activity signature function viewed as a function of $p$ is then given by the ratio between two realized power variations (at two different frequencies):

$$
b_{t}(p)=\frac{\ln (2) p}{\ln (2)+\ln \left[V\left(p, 2 \Delta_{n}\right)\right]-\ln \left[V\left(p, \Delta_{n}\right)\right]}
$$

In practice, the domain $p \in(0,4]$ exhausts the relevant information in the observed $x_{i}$. The reason behind choosing such an interval is that the activity index, which is calculated from power variations, has a discriminant behavior for low powers $p$, in particular around 2. Examining large powers $(p>4)$ does not help in selecting among the different stochastic processes, as at this power level the large jumps mainly matter. This is also the case for $p>2$ (so that the interval $[2,4]$ is sufficient to examine the presence of large jumps). For high-frequency data with $T$ periods $(t=1,2, \ldots, T)$, it should be possible to compute the activity signature function for each of the $T$ intervals over a fine grid of $p$, yielding $\left\{\left\{b_{t}(p)\right\}_{p \in(0,4]}\right\}_{t=1}^{T}$.

The activity index is always in the interval $[0,2]$, at least when large jumps are not considered. For an absolutely continuous process, the index is always 1 . If the estimated activity index is in the interval $(0,1)$, it means that the observed process is from a pure-jump model with no drift and with jumps having infinite variation. If the estimated index is in the interval $(1,2)$, then the appropriate model for the observed process is again a pure-jump model, but with jumps exhibiting infinite variation. If the estimated activity is 2 , then a continuous martingale is indicated.

Todorov and Tauchen (2010) have constructed a test for the presence of a continuous component against the alternative of a pure-jump process by using the activity signature function evaluated 
at a fixed power. ${ }^{19}$ We follow thoroughly their approach in our empirical application to carbon futures.

\subsection{Data}

We use high-frequency data on the EUA Futures contract traded on the European Climate Exchange (ECX) for the period going from January 2, 2009 to June 15, 2010 (369 trading days). Our data set includes transaction prices (as opposed to quotes), i.e. tick-by-tick data, with time stamps for the 2009 futures contract until the end of November 2009, and then for the 2010 futures contract. $^{20}$ This type of data for the EUA ECX market has been studied in Chevallier and Sévi (2010, 2011), where distributional properties with/without jumps are investigated. Each trading day is 11-hour, and sampling every five-minute gives 132 log-returns (increments): these are intraday returns as opposed to daily returns that are more commonly used in financial applications. These intraday returns are used to compute power variation as presented in Eq.(2).

Tick data from ECX are not 'clean' in the sense that a large number of errors (such as zeroprice trades or transactions with an abnormal price) is present. We use the filter rules suggested in Barndorff-Nielsen et al. (2009, Section 3) to clean the dataset. More precisely:

1. We delete entries with a time stamp outside the trading session.

2. We delete entries with a transaction price equal to zero.

3. We delete entries with corrected trades or trades associated with an abnormal reported code.

4. We use the median price when several transactions have the same time stamp.

5. We delete entries that have prices which may be considered as anomalies $(+$ or $-2 \%$ compared with the previous and the next price).

The cleaning procedure allows to evaluate the quality of the data, which is found to be quite good with only 22,177 observations that have been removed (or $4.8 \%$ of the original dataset). We end with a total of 437,801 tick-by-tick observations for 369 trading days which is equivalent to an average 1,187 transactions per day in the ECX futures market. ${ }^{21}$ To illustrate this data, the middle panel of Figure 1 reports the daily number of transactions for the January 2009-June 2010 period. The liquidity appears to be slightly increasing with only a slowdown in the end of 2009

\footnotetext{
${ }^{19}$ Details on the setting of the test and the calculation of standard errors are not detailed here to conserve space, and can be found in Todorov and Tauchen (2010).

${ }^{20}$ In other words, our dataset includes all transactions on the front-year contract, i.e. all prices where two investors have agreed to take simultaneously a long and a short position, respectively (Kolb and Overdahl (2006) is an excellent reference for an introduction to futures markets).

${ }^{21}$ A similar analysis would not have been possible in the BlueNext spot market for $\mathrm{CO}_{2}$ allowances in light of its insufficient liquidity, which yields to unreliable estimates (only 38,924 ticks are available during our sample period in BlueNext).
} 
where trades are shared between the 2009 and the 2010 contract. With one transaction every 33.38 seconds, our estimates using intraday returns will indeed be reliable. ${ }^{22}$

The EUA ECX futures price series for the period under investigation is plotted in the top panel of Figure 1. Large moves can be observed in the allowance futures price, in particular during the January-March 2009 period which corresponds to a period of transmission of the financial crisis to the carbon market (Chevallier (2011)).

The bottom panel of Figure 1 plots the daily jump part that is computed from the methodology developed in Barndorff-Nielsen and Shephard (2006). The jump part is estimated as the noncontinuous part of the intradaily variations by using asymptotic theory to detect changes that are too large to be the likely consequence of a Brownian motion (the methodology is presented in Chevallier and Sévi (2010)). We observe a cluster of jumps with the highest intensity around the period of large allowance price movements in January-March 2009. This graphical inspection indicates that jumps should be found in our investigation by using the ASF methodology, but it is not indicative about the nature of the jumps that are likely to be found (jump intensity and activity) or the presence of a continuous component in the price process.

${ }^{22}$ This was 50 seconds between each transaction for the 2008 futures contract studied in Chevallier and Sévi (2010, 2011). However, the authors empirically show that this is sufficient to use intraday returns to compute, say, the realized volatility, and that the estimates are not too noisy. 
Figure 1: EUA futures price series for the ECX market (top), number of transactions per day (ticks) (middle) and daily jump part (bottom)
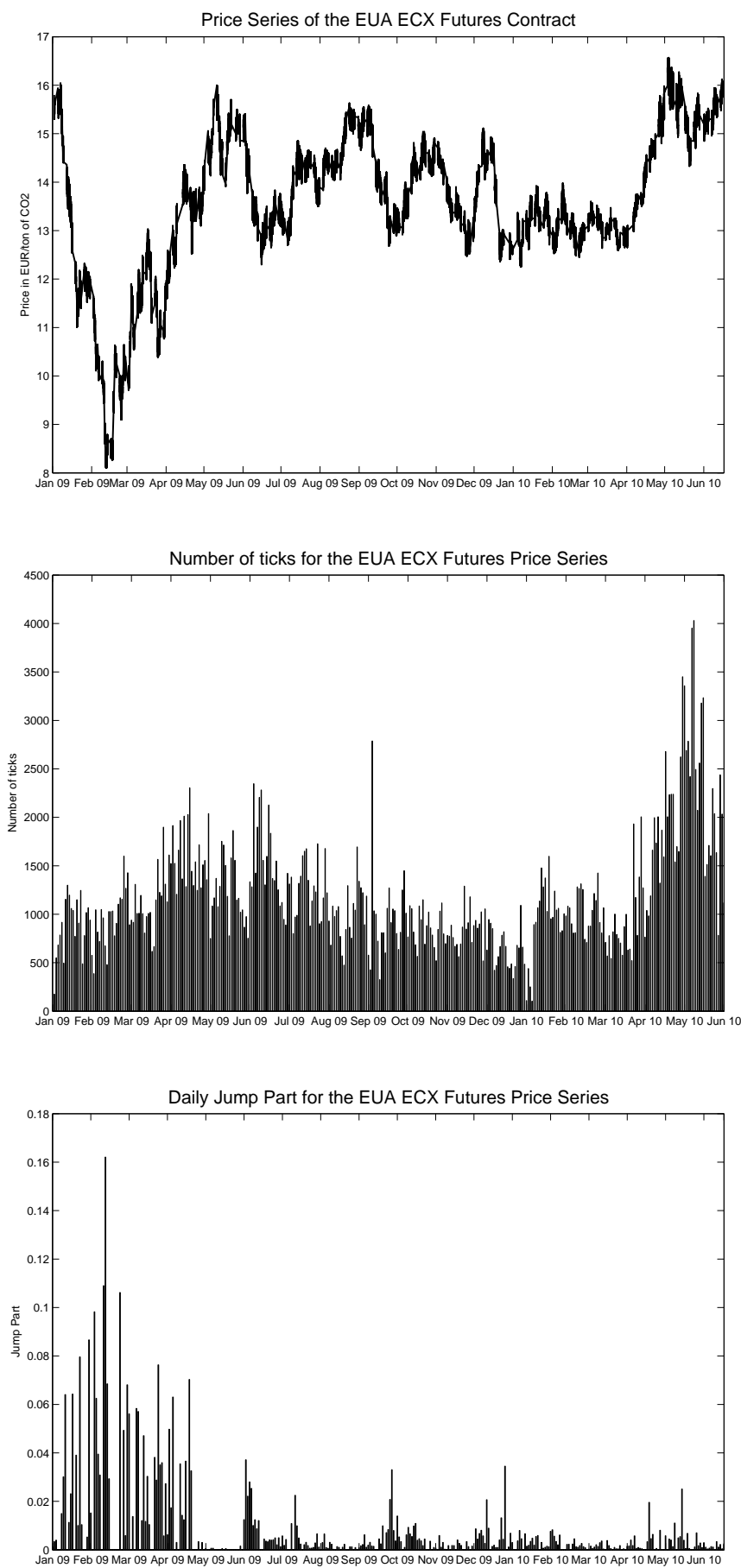

Source: European Climate Exchange 


\subsection{Results}

For each day, we first compute the power variation $V\left(p, \Delta_{n}\right)$ as in Eq.(2) by using 5-minute sampling frequency where the power $p \in(0,4]$ ranges over a grid with 0.01 increments. In our empirical application, we enhance the genuine methodology in Todorov and Tauchen (2010) by relying on the two-time scales principle as in Zhang et al. (2005). ${ }^{23}$ This simple improvement, however, is of interest in our context where the carbon futures contract in the ECX market is less liquid that standard assets such as foreign exchange securities or stocks, among others. We then calculate the power variation $V\left(p, 2 \Delta_{n}\right)$, i.e. with a 10-minute sampling interval. By using the computed power variations over the two sampling frequencies, we compute the ASF $b_{t}(p)$ as in Eq.(3). We thus end with 369 ASFs, which correspond to the number of trading days in our sample. To report intelligible results, we use empirical quantiles of these 369 ASFs. We follow Todorov and Tauchen (2010) who advocate to look only for measures of central tendency and dispersion of the signature functions to draw some conclusions about the general behavior of the activity index. Precisely, define the Quantile Activity Signature Function (QASF) as:

$$
b_{\alpha}(p)=\text { Quantile }_{\alpha}\left[\left\{b_{t}(p)\right\}_{t=1}^{N}\right], \quad \text { for each } p \in(0,4]
$$

with Quantile $\alpha$ the $\alpha$ th quantile of the sequence of $b_{t}(p)$. Very informative plots are obtained from the lower $b_{0.25}(p)$ and upper $b_{0.75}(p)$ quartiles, and the median $b_{0.50}(p)$ over the range $0 \leq$ $p \leq 4$. As it will be seen below, using statistically robust measures such as quantiles limits the impact of extreme observations on the reported ASFs.

Our main result is presented in Figure 2 which shows the QASF for $\alpha=0.25,0.50,0.75$ computed from intraday returns. For robustness checks, the power variations are computed by using intraday returns over either 1-day or 5-day intervals. ${ }^{24}$ The inspection of Figure 2 is indicative of the stochastic characteristics of the price process for carbon futures contracts. The abscissa represents the values of $p$. The ordinates represent the heights of $b(p)$. To fix ideas, if one were in the presence of a process containing a continuous (Brownian) component, the median (and possibly the other quartiles) should stay flat around 2 over the entire domain $p \in(0,4]$. Indeed, this is not the case and we can unambiguously reject the null hypothesis of a Brownian motion in carbon futures prices (at least for the period under consideration). In what follows, a formal test will confirm this preliminary finding.

For abscissa near zero, the activity index is very low, indicating the possible absence of a drift, whose activity index is 1.0. For larger values of $p$, the three QASFs increase linearly as they would do in the case where jumps are dominating the activity index. In particular, for abscissa above

\footnotetext{
${ }^{23}$ We keep a sampling interval for intraday returns of 5-minute as in the bulk of the literature when using this type of data, but we sample with starting points at each minute. Thus, we have 5 more estimates of the ratio at each period, and an average of these ratios provides much more robust results.

${ }^{24}$ Despite the fact that the computation of power variations relies on infill asymptotics (i.e. the interval is sampled over a finer and finer mesh as the sample size increases), less noisy estimates can obviously be obtained by using more data. Todorov and Tauchen (2011) suggest a 22-day block which is a good 'compromise in the tradeoff between the presumption of constant activity over the subinterval and the associated reduction in sampling error inference with more data points per interval'. (p. 362). Due to data limitation, we experiment with 5-day blocks in our empirical analysis.
} 
2 , only jumps matter asymptotically. Hence, for $p>2$, the behavior of the activity signature function can reveal us whether there are jumps, large or small, in the process. The upper $b_{0.75}(p)$ and lower $b_{0.25}(p)$ QASFs provide an indication of sampling dispersion. More precisely, the $b_{0.75}(p)$ QASF provides evidence of large jumps that only occur occasionally as the dotted line is at a good distance from the median QASF. This highlights the need for using quartiles that are robust to extreme observations, but also indicates that large and infrequent jumps should be part of the stochastic modeling of carbon prices. The lower $b_{0.25}(p)$ QASF shows that most of the days, the hypothesis of the presence of a continuous martingale can be left aside (as it will be shown in the formal test at the end of this Section). Overall, our results suggest that the process generating the carbon futures price data lacks a continuous component, as it deviates strongly from the reference horizontal line set at 2. Based on our dataset, we can conclude that the process is a pure-jump process of infinite activity. The large dispersion between all the ASFs, highlighted by the large distance between the upper $b_{0.75}(p)$ and lower $b_{0.25}(p)$ QASFs, reflects the high intensity of the jumps during some days. This lead to consider as a potential candidate a mixture of pure-jump model with a second jump model having infrequent but large jumps.

Similar comments arise from the bottom panel of Figure 2, i.e. with 5-day blocks, which can be seen as a sensitivity test to the choice of the length of the block. The median QASF here is more 'erratic', as the large jumps have an impact on a larger proportion of blocks. Nevertheless, the alteration of the ASFs computed over 5-day blocks is limited, and our qualitative results remain valid.

As an additional analysis to the empirical results given above, we present graphically the point estimates $\log _{b_{s}}(p)$ for $p=0.90$. The value for $p$ is chosen according to the theoretical developments in Todorov and Tauchen (2010), who show that a value near one is an optimal choice. The idea is to compare the day-by-day values of $\log _{b_{s}}(p)$ with the null value of $\log (2)=0.69$. Figure 3 plots the computed values for $\log _{b_{s}}(p)$ with $p=0.90$ for our full sample. As it can be observed from the plot, no point estimate lies above the 0.69 threshold thereby confirming the absence of a continuous martingale. ${ }^{25}$

Then, we report in Table 2 some descriptive statistics about the estimated activity index for different values of $p$. Column (2) in the Table reports the median of the point estimates of the activity index, along with the median absolute deviation about the median (MAD $=$ med $\mid \hat{\beta}$ $\operatorname{med}(\hat{\beta}) \mid)$ in column $(3)$. The statistics indicate that the activity index for carbon futures is in the range of 1.82 for values of $p$ below 0.50 . Again, this result implies the absence of a continuous component in the stochastic process as the continuous component should have an impact at all values of $p$. Our results are not directly comparable with those of Todorov and Tauchen (2011) who use higher values of $p$ that are more discriminant in their framework.

Finally, as stated in Section 3.1, an activity level of 2 has a special meaning since it separates pure-jump models from models containing continuous martingales. In the last column of Table 2,

\footnotetext{
${ }^{25}$ Note that some point estimates $\log _{b_{s}}(p)$ may lie above the 0.69 line, as long as they are not too significantly above the line. A statistical threshold has normally to be computed from the Theorem 2 in Todorov and Tauchen (2010). As all our point estimates are below 0.69, we do not need to investigate further confidence bounds issues.
} 
Figure 2: QASF for the EUA ECX Futures Price Series for 1-day blocks (top), and 5-day blocks (bottom)
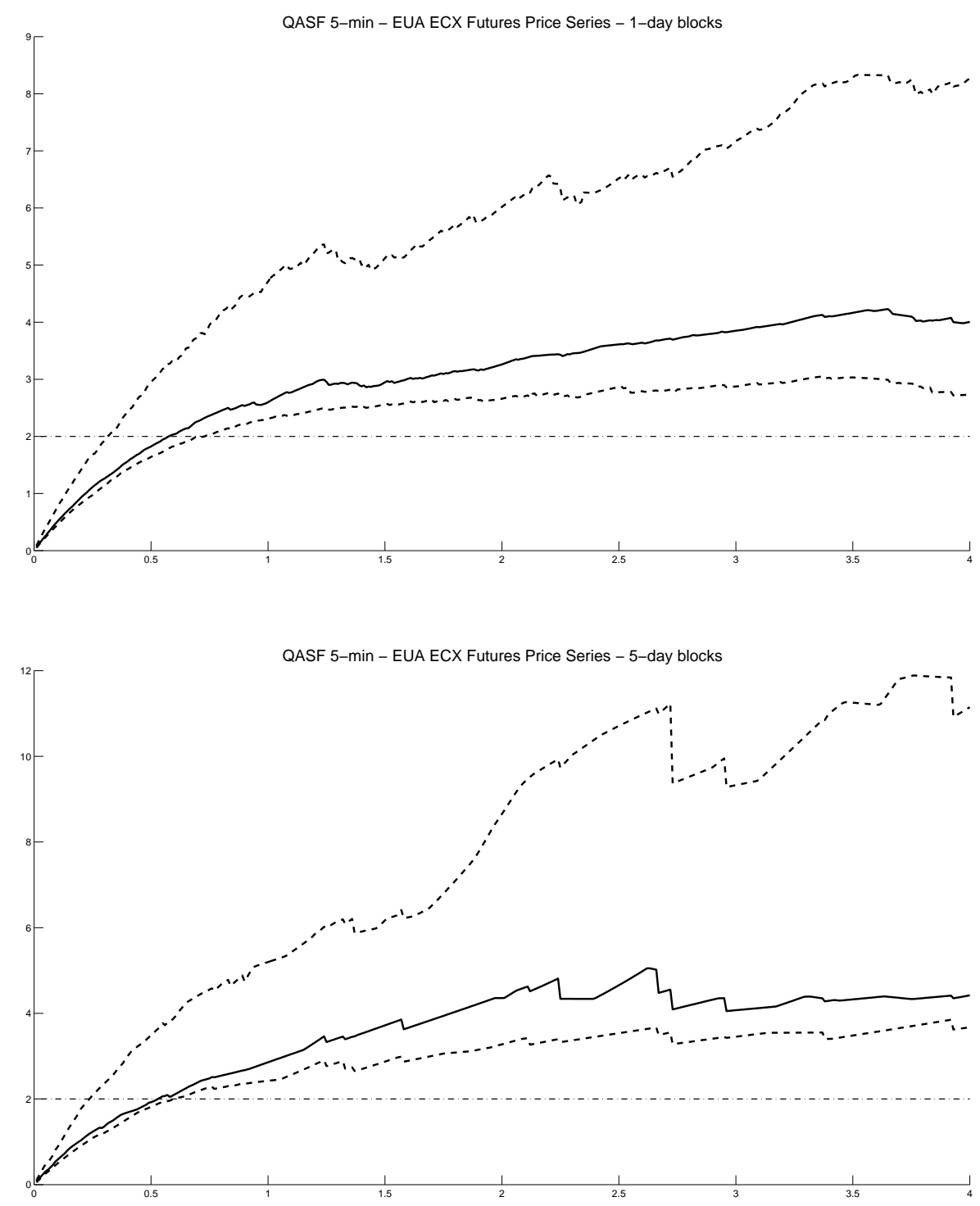

Note: QASF is computed using 5-minute sampling intervals. Lower and upper-quartiles are represented in dashed lines, along with the reference horizontal line fixed at 2 . 
Figure 3: Daily estimates of $\log _{b_{s}(p)}$

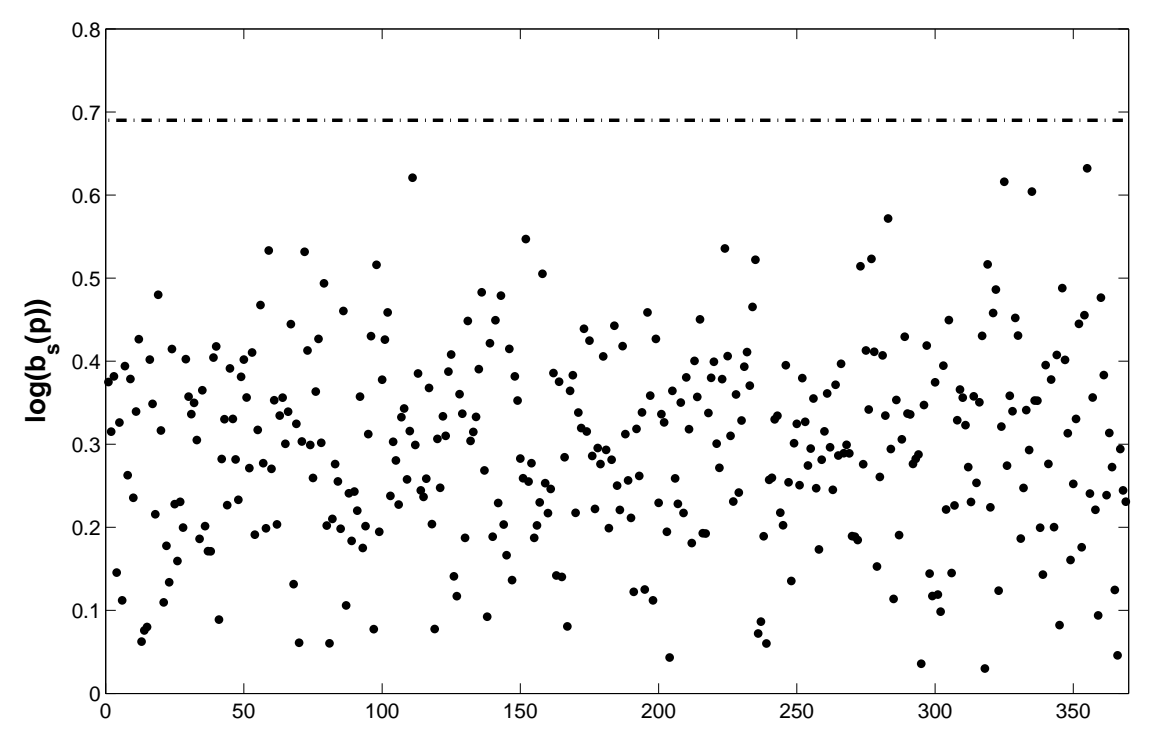

$\underline{\text { Note: }}$ Daily point estimates $\log _{b_{s}(p)}$ marked by $\bullet$ for $p=0.90$. A point estimate below its corresponding confidence bound would be considered statistically significantly different from the null value of $\log (2)=0.69$ 
we conduct a test, following the Theorem 2 in Todorov and Tauchen (2010), whether the estimated activity index is statistically less than 2 (the test is one-sided). The test is qualitatively equivalent to the tests of Aït-Sahalia and Jacod (2010), Jing et al. (2012) or Kong (2012). Recall that these tests allow to check formally the presence (or alternatively the absence) of a Brownian motion in the underlying process. The null hypothesis is that a continuous martingale component is present in the process. The alternative is that the process is a pure-jump model (i.e. that it lacks a continuous component). The test is conducted for different values of $p$. The rejection rates are between 0.45 and 0.98 at the $5 \%$ level. Hence, we find strong statistical evidence against a model with a continuous component.

Table 2: Test for presence of Brownian component in EUA ECX Futures Price Series

\begin{tabular}{cccc}
\hline$p$ & $\operatorname{med}(\hat{\beta})$ & MAD & Rejections $(5 \%)$ \\
\hline 0.10 & 0.5099 & 0.1702 & 98.04 \\
0.20 & 0.9258 & 0.2907 & 89.10 \\
0.30 & 1.2588 & 0.3884 & 78.60 \\
0.40 & 1.5611 & 0.4668 & 62.80 \\
0.50 & 1.8234 & 0.5611 & 45.30 \\
\hline
\end{tabular}

Note: MAD stands for the median absolute deviation about the median.

Overall, our empirical results indicate that all the stochastic processes that have been proposed in the literature so far (see Section 2) are not plausible in light of the analysis of the high-frequency data. The evidence in Figures 2 and 3 strongly invalidates models including a continuous martingale. Similar to the analysis of the VIX in Todorov and Tauchen (2011) or Internet data in Todorov and Tauchen (2010), our results show that the carbon futures price data for the period under investigation cannot be generated from a stochastic process including a Brownian motion (as the activity index is not flat at all or near to be flat around a value of 2).

\subsection{Sensitivity analysis}

The sensitivity of the empirical results with respect to our modeling choices is now examined. The ASF methodology can be modified in several dimensions. In the previous section, we have already robustified the initial approach by using the two-time scales as in Zhang et al. (2005). In addition, we have checked for the sensitivity of our results to the number of consecutive days (block) used to compute $b(p)$. We cannot cut our sample in two or more different sub-samples as we are limited by the shortness of our original time sample. Another possible sensitivity test of our results can arise from the chosen frequency to create a discrete time grid for sampling the transaction data.

In Figure 4, we report the QASF for 2.5 and 10 minutes as alternatives to the 5 -min choice used in the previous sub-Section. ${ }^{26}$ We provide graphical results for both 1-day and 5-day blocks. We observe that using alternative sampling frequencies does not alter our main result. Qualitatively,

\footnotetext{
${ }^{26}$ The sampling frequency is known to play a major role when using tick-by-tick data because of the microstructure noise in observed prices. As for the carbon price, see the discussion in Chevallier and Sévi (2010, 2011). Jing et al. (2011) develop theoretical results about the estimation of the activity index in a noisy context.
} 
Figure 4: QASF for the EUA ECX Futures Price Series for 1-day blocks (top), and 5-day blocks (bottom)
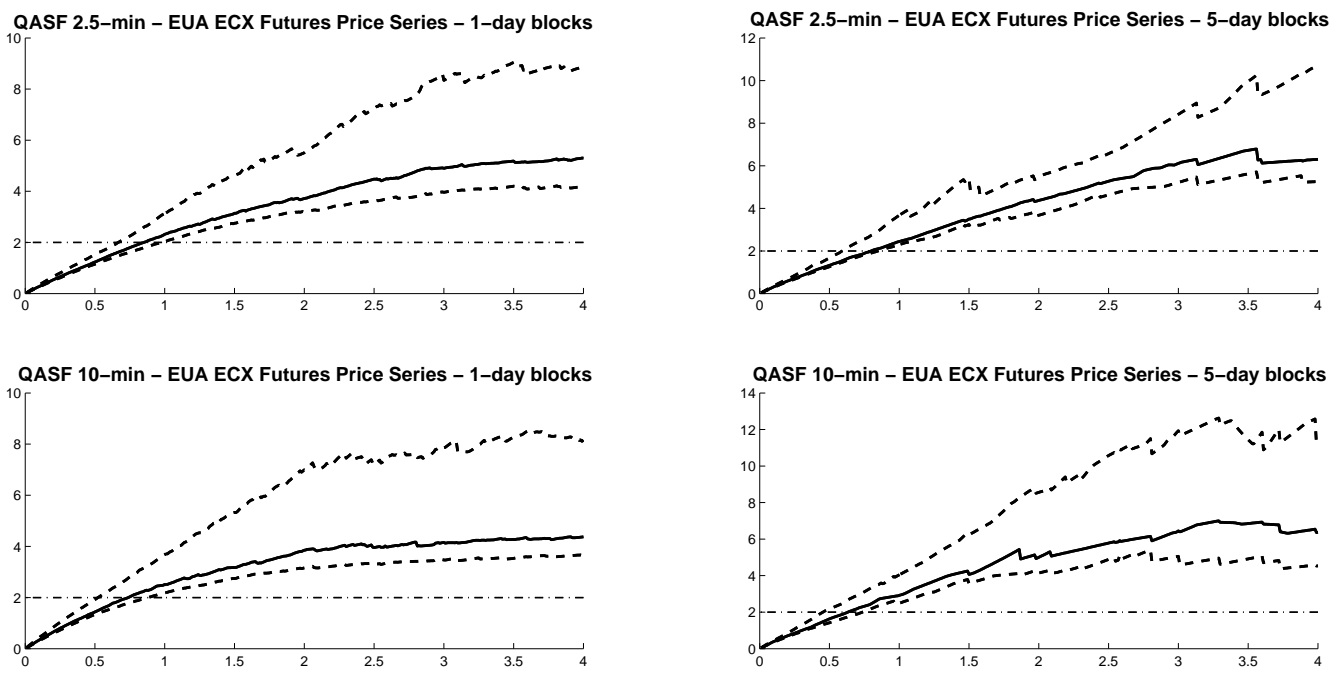

Note: QASF is computed using 2.5 or 10-minute sampling intervals with Lower and upper-quartiles are represented in dashed lines, along with the reference horizontal line fixed at 2 .

the four plotted QASF still indicate the absence of a Brownian motion, as well as the presence of a pure-jump process with less frequent (possibly large) additional jumps. In short, we are able to confirm our findings that the carbon futures price would be adequately modelled by using a mixture of a pure-jump model with a Poisson- or Lévy-like jump process. 


\subsection{Discussion: On the origins and economic consequences of jumps on tradable permits markets}

To sum up, the jumps identified in our empirical analysis can be of two categories: (i) small jumps of infinite activity, and (ii) large (but infrequent) jumps.

Concerning the former category, we suggest an interpretation of our empirical findings, whereby a pure-jump process may be an adequate tool to model carbon prices. Our explanation relies on the theoretical argument by Pakkanen (2010) that only a large number of agents do lead to a diffusion (i.e. Brownian motion) on a financial market. When only a limited number of investors act on the market place, prices only move when orders are executed thereby supporting the view of a pure-jump as an adequate process in this case. As such, it appears that the lack of liquidity may explain the absence of a continuous component in the carbon price so far.

Concerning the latter category, the mathematical papers by previous literature (especially Dannenberg and Ehrenfeld (2011), Çetin and Verschuere (2009), Borovkov et al. (2011)) have derived smart modeling strategies to model the importance of jumps in the price process of $\mathrm{CO}_{2}$ (see the Review section 2).

These jumps arise due to political uncertainties on the carbon markets, whereby the European Commission intervenes on the emissions trading system to change the allocation rules (and the amount of allowances distributed each year). They could also correspond to changes in relative fuel prices (oil, gas, coal), which have been shown to impact periodically the variation of carbon prices.

Therefore, we believe that our paper contains a contribution relative to the environmental economics literature, which is to stress the importance of modeling jumps in the stochastic process of carbon futures prices.

This feature has been largely ignored in previous environmental economics literature, and has been made obvious thanks to our empirical diagnostic tool based on the activity signature function of the $\mathrm{CO}_{2}$ price process.

Hence, we attempt to build a bridge between these two separate literatures, and to link our empirical finding to the understanding of how international emissions trading schemes work in practice. Ignoring these numerous interventions from the environmental regulator in the governance of the ETS would indeed be detrimental to the quality of the price forecasts produced by market analysts and economists on this environmental market.

The contribution of our paper is also to shed more light on the likely origin for these jumps, i.e. due to political decisions or to the relative fluctuation of energy prices.

Another important aspect is that, following jumps, agents can revise their expectations about emissions or price levels (depending on the stringency of the cap, the level of economic activity or the current levels of fuel prices). Our study therefore allows to point out that jumps play a central role in the formation of anticipations of rational economic agents on the European emissions trading system, and their reaction to any kind of news relevant to the formation of the $\mathrm{CO}_{2}$ price process. This characteristic of market efficiency is a desirable property for any newly created environmental 
market (exchangeable allowances of $\mathrm{CO}_{2}$ being a 'currency' in this setting).

\section{Conclusion}

Following the review of previous literature on the stochastic properties of carbon prices, our empirical application based on the activity signature function reveals that a pure-jump model is appropriate for the modeling of carbon futures. We find that the process is very vibrant. Hence, a good stochastic model could be one of the pure-jump type, whose driving jumps come from a very active Lévy process with possibly large jumps coming from a Poisson process.

These findings lead to several economically important conclusions. First, large jumps (coming from institutional news releases, energy or macroeconomic markets shocks) need to be explicitly taken into account when modelling spot and futures carbon price series. Second, the pure-jump dynamics of the process would imply that hedging is quite complicated on the carbon market, since volatility risk cannot be fully spanned with derivatives instruments.

Documenting the absence of a Brownian motion in the underlying stochastic process for the carbon price does not only have an interest on its own, but also for practical applications such as derivatives pricing, investment analysis or hedging behavior. As emphasized in Aitt-Sahalia and Jacod (2009a,b), the presence of jumps and/or the absence of a continuous martingale also has important implications for portfolio choice or risk management activities. As many economic agents have significant positions in the carbon financial market, they face an important price risk that does depend on the stochastic properties of the carbon price process. We show that, in the context of a Value-at-Risk (VaR) analysis which is common practice for large firms nowadays, the risk is not well estimated by using the processes suggested in the literature so far, and efforts should be made to accommodate risk exposure according to more plausible processes. The absence of a continuous martingale suggests that models such as the Barndorff-Nielsen and Shephard (2001) or the CGMY (2002) pure-jump models are likely candidates. ${ }^{27}$ Other less well-known potential candidates are listed in the contribution by Jing et al. (2012).

We can also explain the gist of our results thanks to Pakkanen (2010), who states that a high number of agents on a given market creates the condition for modelling price processes with a Brownian motion. Conversely, when the liquidity is lower, the smaller number of interactions between agents implies that the price process can be better approximated with a pure-jump model. Based on our analysis of the intraday data, the $\mathrm{CO}_{2}$ markets can certainly be considered as less liquid than other financial markets (compared to foreign exchange markets as a benchmark for instance). There are therefore less interactions, and the corresponding price process does not contain a continuous component. In future work, it could be interesting to extend our results to the 2010-2012 period. Most likely, we could presume that the same kind of conclusion would still hold against the background of the financial crisis (and associated low volumes of trades).

\footnotetext{
${ }^{27}$ See also a discussion on the implications of these pure-jump models in the Appendix.
} 


\section{References}

AïT-Sahalia, Y., Jacod, J., 2009a. Testing for jumps in a discretely observed process. Annals of Statistics 37, 184-222.

AїT-Sahalia, Y., JACOD, J., 2009b. Estimating the degree of activity of jumps in high frequency financial data. Annals of Statistics 37, 2202-2244.

AїT-SAhalia, Y., JACOD, J., 2010. Is Brownian motion necessary to model high-frequency data? Annals of Statistics 38, 3093-3128.

BALlotTA, L., 2005. A Lévy process-based framework for the fair valuation of participating life insurance contracts. Insurance: Mathematics and Economics 37, 173-196.

Barndorff-Nielsen, O., Shephard, N., 2001. Non-Gaussian Ornstein -Uhlenbeck-based models and some of their uses in financial economics. Journal of the Royal Statistical Society Series B $63,167-241$.

Barndorff-Nielsen, O., Shephard, N., 2004. Power and bipower variation with stochastic volatility and jumps. Journal of Financial Econometrics 2, 1-37.

Barndorff-Nielsen, O., Shephard, N., 2006. Econometrics of testing for jumps in financial economics using bipower variation. Journal of Financial Econometrics 4, 1-30.

Barndorff-Nielsen, O.E., Hansen, P.R., Lunde, A., Shephard, N., 2009. Realized kernels in practice: trades and quotes. Econometrics Journal 12, C1-C32.

Barndorff-Nielsen, O., Shephard, N., 2012. Lévy Driven Volatility Models, Oxford University Press, forthcoming.

Benz, E., TrüCK, S., 2009. Modeling the price dynamics of $\mathrm{CO}_{2}$ emission allowances. Energy Economics 31, 4-15.

Blumenthal, R., Getoor, R., 1961. Sample functions of stochastic processes with independent increments. Journal of Mathematics and Mechanics 10, 493-516.

Borovkov, K., Decrouez, G., Hinz, J., 2011. Jump-diffusion modeling in emission markets. Stochastic Models 27, 50-76.

Carmona, R., Hinz, J., 2011. Risk-Neutral Models for Emission Allowance Prices and Option Valuation. Management Science 57, 1453-1468.

Carmona, R., Fehr, M., Hinz, J., 2009. Optimal stochastic control and carbon price formation. SIAM Journal on Control and Optimization 48, 2168-2190.

Carmona, R., Fehr, M., Hinz, J., Porchet, A., 2010. Market designs for emissions trading schemes. SIAM Review 52, 403-452.

Carr, P., Geman, H., Madan, D.B., Yor, M., 2003. Stochastic Volatility for Lévy Processes. Mathematical Finance 13, 345-382. 
Carr, P., Madan, D.B., 1999. Option Valuation Using the Fast Fourier Transforms. Journal of Computational Finance 2, 61-73.

Çetin, U., Verschuere, M., 2009. Pricing and Hedging in Carbon Emissions Markets. International Journal of Theoretical and Applied Finance 12, 949-967.

Chesney, M., Taschini, L., 2012. The Endogenous Price Dynamics of Emission Allowance and An Application to CO2 Option Pricing. Applied Mathematical Finance, forthcoming. doi:0.1080/1350486X.2011.639948

Chevallier, J., 2011. A model of carbon price interactions with macroeconomic and energy dynamics. Energy Economics 33, 1295-1312.

Chevallier, J., SÉvi, B., 2010. Jump-robust estimation of realized volatility in the EU emissions trading scheme. Journal of Energy Markets 3, 49-67.

Chevallier, J., Sévi, B., 2011. On the Realized Volatility of the ECX $\mathrm{CO}_{2}$ Emissions 2008 Futures Contract: Distribution, Dynamics, and Forecasting. Annals of Finance 7, 1-29.

Cont, R., Tankov, P., 2004. Financial Modelling with Jump Processes. Chapman \& Hall/CRC, Boca Raton, Florida, USA.

Cronshaw, M.B., Kruse, J.B., 1996. Regulated Firms in Pollution Permit Markets with Banking Journal of Regulatory Economics 9, 179-189.

Cvitanić, J., Polimenis, V., Zapatero, F., 2008. Optimal portfolio allocation with higher moments. Annals of Finance 4, 1-28.

Dales, J.H., 1968. Pollution, Property and Prices. Toronto University Press, Canada.

Dannenberg, H., Ehrenfeld, W., 2011. A Model for the Valuation of Carbon Price Risk. In Emissions Trading: Institutional Design, Decision Making and Corporate Strategies, eds Antes, R., Hansjürgens, B., Letmathe, P., Pickl, S., Springer, Germany.

Daskalakis, G., Psychoyios, D., Markellos, R.N., 2009. Modeling $\mathrm{CO}_{2}$ emission allowance prices and derivatives: Evidence from the European trading scheme. Journal of Banking and Finance $33,1230-1241$.

Ellerman, A.D., 2005. A note on tradeable permits. Environmental and Resource Economics $31,123-131$.

Ellerman, A.D., Buchner, B.K., 2008. Over-Allocation or Abatement? A Preliminary Analysis of the EU ETS Based on the 2005-06 Emissions Data. Environmental and Resource Economics $41,267-287$.

Feldman, R.M, Valdez-Flores, C., 2010. Applied Probability and Stochastic Processes. Springer, Heidelberg, Germany. 
Ghahramani, S., 2005. Fundamentals of Probability with Stochastic Processes. Pearson Education, Prentice Hall, New Jersey, USA.

Gillespie, D.T., 1996. Exact numerical simulation of the Ornstein-Uhlenbeck process and its integral. Physical Review E 54, 2084-2091.

Hanson, F.B., Westman, J.J., 2002. Portfolio Optimization with Jump-Diffusions: Estimation of Time-Dependent Parameters and Application. Proceedings of the $41^{\text {st }}$ IEEE Conference on Decision and Control, 377-381.

Hanson, F.B., 2007. Applied Stochastic Processes and Control for Jump-Diffusions: Modeling, Analysis and Computation. SIAM Press, Society for Industrial and Applied Mathematics, Philadelphia, USA.

Hinz, J., Novikov, A., 2010. On fair pricing of emission-related derivatives. Bernoulli 16(4), $1240-1261$.

JING, B.-Y., Kong, X.-B., LIU, Z., 2011. Estimating the jump activity index under noisy observations using high-frequency data. Journal of the American Statistical Association 106, 558-568.

Jing, B.-Y., Kong, X.-B., LiU, Z., 2012. Modeling high-frequency financial data by pure jump processes. Annals of Statistics 40, 759-784.

Kannan, D., 1979. An Introduction to Stochastic Processes. North Holland Series in Probability and Applied Mathematics, New York, USA.

Kassberg, S., Kiesel, R., Liebmann, T., 2008. Fair valuation of insurance contracts under Lévy process specifications. Insurance: Mathematics and Economics 42, 419-433.

Kling, C., Rubin, J., 1997. Bankable permits for the control of environmental pollution. Journal of Public Economics 64, 101-115.

Knill, O., 2009. Probability and Stochastic Processes with Applications. Overseas India Press, New Delhi, India.

Kolb, R.W., Overdahl, J.A., 2006. Understanding Futures Markets, Blackwell Publishing, New York.

KonG, X.-B., 2012. Is a pure jump process fitting the high frequency data better than a jumpdiffusion process? Journal of Statistical Planning and Inference, forthcoming.

Kroese, D.P., Taimre, T., Botev, Z.I., 2011. Handbook of Monte Carlo Methods. Wiley Series in Probabilities and Statistics, Chichester, UK.

LEIBY, P., Rubin, J., 2001. Intertemporal permit trading for the control of greenhouse gas emissions. Environmental and Resource Economics 19, 229-256.

Lin, Y.N., Lin, A.Y., 2007. Pricing the Cost of Carbon Dioxide Emission Allowance Futures. Review of Futures Markets 16, 1-16. 
Liu, J., Longstaff, F., Pan, J., 2003. Dynamic asset allocation with event risk. Journal of Finance 58, 231-259.

MaedA, A., 2004. Impact of Banking and Forward Contracts on Tradable Permit Markets. Environmental Economics and Policy Studies 6, 81-102.

Martzoukos, S.H., Trigeorgis, L., 2002. Real (investment) options with multiple sources of rare events. European Journal of Operational Research 136, 696-706.

Montgomery, D.W., 1972. Markets in licenses and efficient pollution control programs. Journal of Economic Theory 5, 395-418.

PAKKANEn, M.S., 2010. Microfoundations for diffusion price processes. Mathematics and Financial Economics 3, 89-114.

Paolella, M.S., Taschini, L., 2008. An econometric analysis of emission allowance prices. Journal of Banking and Finance 32, 2022-2032.

Rubin, J., 1996. A model of intertemporal emission trading, banking, and borrowing. Journal of Environmental Economics and Management 31, 269-286.

Schennach S.M., 2000. The Economics of Pollution Permit Banking in the Context of Title IV of the 1990 Clean Air Act Amendments. Journal of Environmental Economics and Management 40, 189-210.

Schoutens, W., 2003. Lévy Processes in Finance. Wiley Series in Probability and Statistics, New York, USA.

SChWARTZ, E.S., 1997. The stochastic behavior of commodity prices: Implications for valuation and hedging. Journal of Finance 52, 923-973.

Schwartz, E.S., Smith, J.E., 2000. Short-term variations and long-term dynamics in commodity prices. Management Science 46, 893-911.

Seifert, J., Uhrig-Homburg, M., Wagner, M., 2008. Dynamic behavior of $\mathrm{CO}_{2}$ prices. Journal of Environmental Economics and Management 56, 180-194.

Speyer, J.L, Chung, W.H., 2008. Stochastic Processes, Estimation, and Control. SIAM Press, Society for Industrial and Applied Mathematics, Philadelphia, USA.

Stevens, B., Rose, A., 2002. A dynamic analysis of the marketable permits approach to global warming policy: a comparison of spatial and temporal flexibility. Journal of Environmental Economics and Management 44, 45-69.

Stirzaker, D., 2005. Stochastic Processes and Models. Oxford University Press, Oxford, UK.

Rolski, T., Schmidli, H., Schmidt, V., Teugels, J., 1999. Stochastic Processes for Insurance and Finance. Wiley Series in Probabilities and Statistics, Chichester, UK. 
Rydberg, T.H., 1997. The Normal Inverse Gaussian Lévy Process: Simulation and Approximation. Communications in Statistics: Stochastic Models 13, 887-910.

Todorov, V., Tauchen, G., 2010. Activity signature functions for high-frequency data analysis. Journal of Econometrics 154, 125-138.

Todorov, V., Tauchen, G., 2011. Volatility jumps. Journal of Business and Economic Statistics 29, 356-371.

Zhang, L., Mykland, P.A., AÏт-Sahalia, Y., 2005. A tale of two time scales: determining integrated volatility with noisy high frequency data. Journal of the American Statistical Association 100, 1394-1411.

Zhu, Z., Graham, P., Reedman, L., Lo, T., 2009. A scenario-based integrated approach for modeling carbon price risk. Decisions in Economics and Finance 32, 35-48. 


\section{Appendix: Key properties of stochastic processes}

In what follows, we provide a brief overview of the key characteristics of stochastic processes, in continuous and jump diffusion settings. By doing so, we build on the notations by Hanson (2007), Knill (2009) and Kroese et al. (2011).

The interested reader may refer to advanced texts in Kannan (1979), Rolski et al. (1999), Schoutens (2003), Cont and Tankov (2004), Ghahramani (2005), Stirzaker (2005), Speyer and Chung (2008), Feldman et al. (2010), or Barndorff-Nielsen and Shephard (2012).

\subsection{Continuous diffusion}

Let $\Omega$ be a probability space, and let $T \subset \mathbb{R}$ be time. A collection of random variables $X_{t}, t \in T$ with values in $\mathbb{R}$ is called a stochastic process.

If $X_{t}$ takes values in $S=\mathbb{R}^{d}$, it is called a vector-valued stochastic process (but often abbreviates by the name stochastic process too).

If the sample function $X_{t}(\omega)$ is a continuous function of $t$ for almost all $\omega \in \Omega$, then $X_{t}$ is called a continuous stochastic process.

Let us start with the Brownian motion ${ }^{28}$ as a fundamental example of an important stochastic process which does not feature mean reversion.

\subsubsection{Brownian motion}

An $\mathbb{R}^{d}$-valued continuous Gaussian process $X_{t}$ with mean vector $m_{t}=E\left[X_{t}\right]$ and covariance matrix $V(s, t)=\operatorname{Cov}\left(X_{s}, X_{t}\right)=E\left[\left(X_{s}-m_{s}\right) \cdot\left(X_{t}-m_{t}\right)\right]$ is called Brownian motion if for any $0 \leq t_{0}<t_{1}<\cdots<t_{n}$, the random vectors $X_{t_{0}}, X_{t_{i+1}}-X_{t_{i}}$ are independent and the covariance matrix $V$ satisfies $V(s, t)=V(r, r)$, where $r=\min (s, t)$ and $s \rightarrow V(s, s)$. It is called the standard Brownian motion if $m_{t}=0$ for all $t$ and $V(s, t)=\min \{s, t\}$.

A numerical example of a Brownian motion computed for 100 observations, $r=0.02$ with a drift $m_{t}=\sqrt{0.1}$ is pictured in Figure 5 .

Define the process $B_{t}=X([0, t])$. For any sequence $t_{1}, t_{2}, \cdots \in T$, this process has independent increments $B_{t}-B_{t-1}$ and is a Gaussian process. For any $x \in \mathbb{R}$, the process:

$$
X_{t}=x+B_{t}
$$

is called Brownian motion started at $x$.

Furthermore, if $B_{t}$ is a Brownian motion, then $X=f(B, t)$ can be written in differential form as:

$$
d X_{t}=\alpha X_{t} d t+\beta d M_{t}, \quad X_{0}=1
$$

\footnotetext{
${ }^{28}$ The Wiener process is a mathematical idealization of Brownian motion, but often the term Brownian motion is used instead of the term Wiener process.
} 


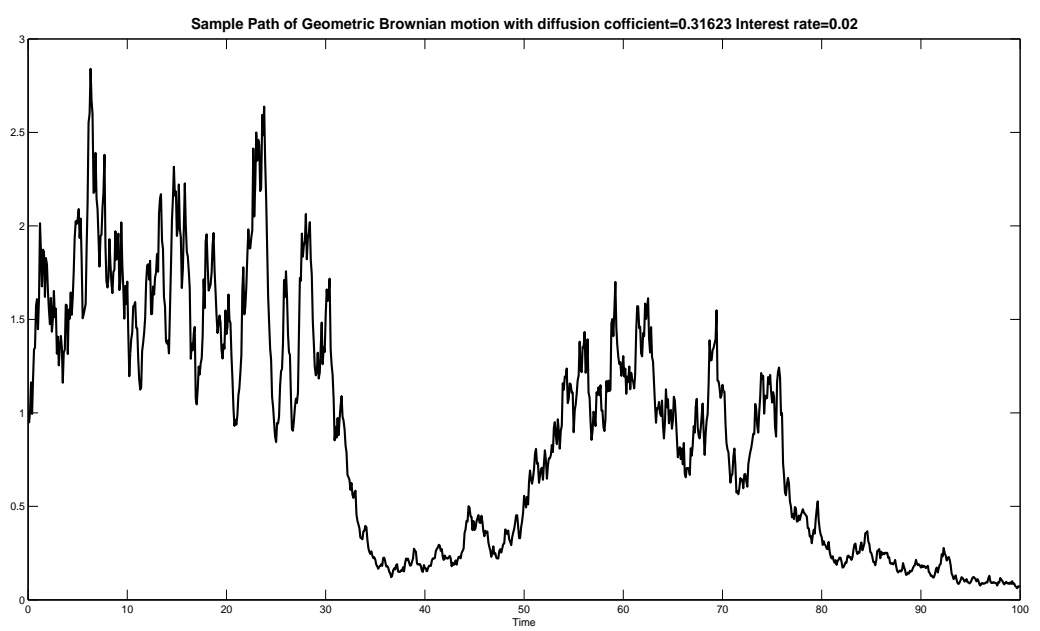

Figure 5: Numerical illustration of Brownian motion

with $\{\alpha, \beta\}$ constants, and $M$ a continuous martingale of finite variation. This is an example of a stochastic differential equation (SDE). Unlike ordinary differential equations, one has to use Ito's formula to integrate when looking for solutions.

Next, we proceed with a generalization of stochastic processes featuring the mean reversion property.

\subsubsection{Ornstein-Uhlenbeck process}

The mean reversion process can be considered as a modification of a random walk, where the alterations to the process do not occur entirely independently.

The Ornstein-Uhlenbeck process has the property that it is mean-reverting, i.e. it always tries to come back to its asymptotic mean value. For this reason, it is also called the oscillatory process. The Brownian motion $B_{t}$ and the Ornstein-Uhlenbeck process $O_{t}$ are for $t \geq 0$ related by:

$$
O_{t}=\frac{1}{\sqrt{2}} e^{-t} B_{e^{2 t}}
$$

The corresponding SDE is obtained by writing:

$$
d X_{t}=-\tau X_{t} d t+\beta d B_{t}
$$

with the parameter $\tau>0$ governing the rate of mean-reversion, and $\beta$ a constant.

An example of the path for the Ornstein-Uhlenbeck process with mean reversion rate $\tau=0.1$ and diffusion constant $\beta=0.03$ is given in Figure 6 . The numerical method used here was published by Gillespie (1996). 


\subsubsection{Vasicek process}

The Vasicek process is very close to the Ornstein-Uhlenbeck process presented above. This process is a diffusion process, which leads to the following closed form formula:

$$
d X_{t}=\kappa\left(\tau-X_{t}\right) d t+\beta d B_{t}
$$

where the parameters $\kappa, \tau$ and $\beta$ are constants, and the random motion is generated by the Brownian motion $B_{t}$. An important property of the Vasicek process is that the mean is reverting to $\tau$, and the tendency to revert is controlled by $\kappa$.

To give an idea of what the Vasicek process looks like, we have generated a sample path in Figure 7 by plugging the values $\tau=0.10, \kappa=0.3$ and $\beta=0.03$. 


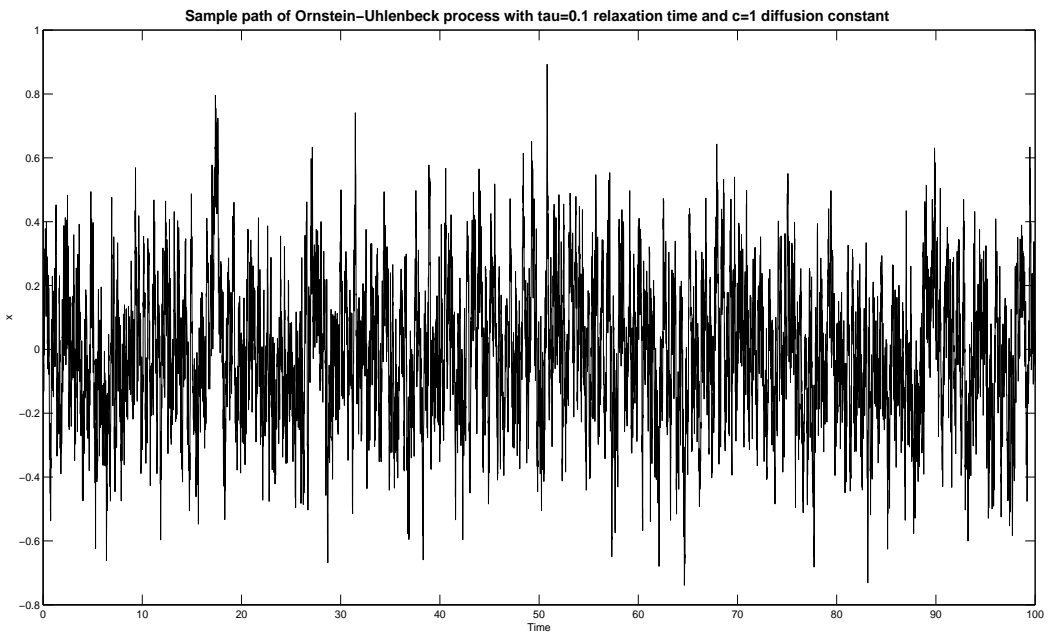

Figure 6: Numerical illustration of Ornstein-Uhlenbeck process

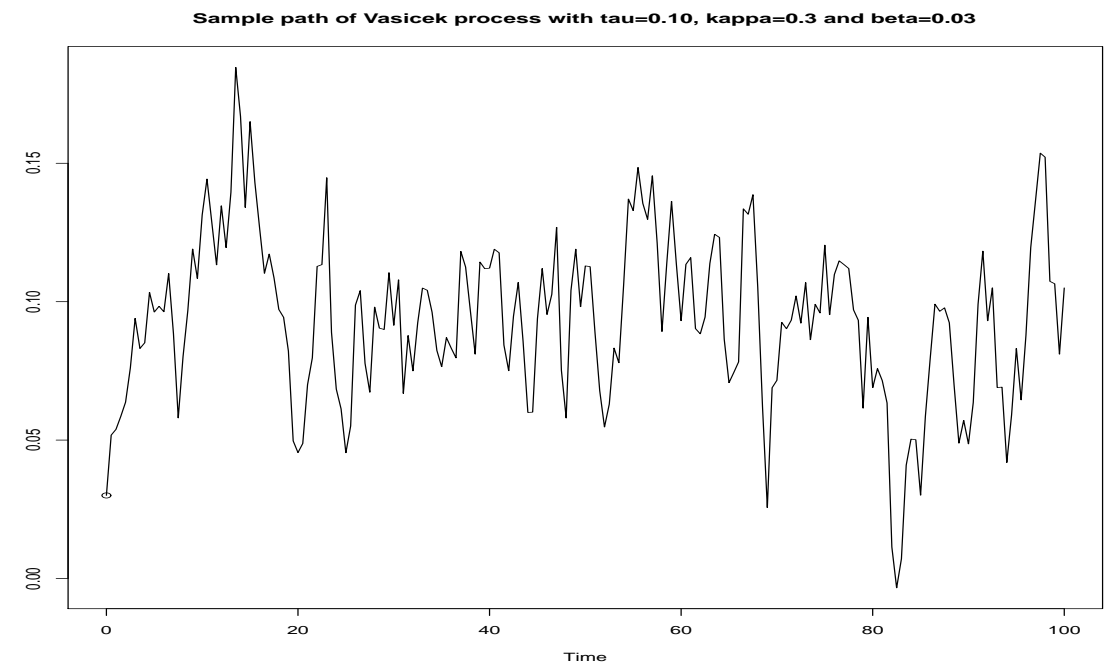

Figure 7: Numerical illustration of Vasicek process 


\section{$5.2 \quad$ Jump diffusion}

In the pure diffusion stochastic model, there is one obvious missing feature that large market fluctuations or crashes / rallies - which characterize the market's bullish or bearish trends - are not represented. These discontinuities highlight the statistical importance of including jumps in financial market models ${ }^{29}$. That is why we present below jump diffusion process models.

\subsubsection{Poisson process}

A Poisson process $P_{t}$ with rate $\lambda$ verifies the following property:

$$
P_{t}=\text { Number of occurrences in }[0, t) \sim P_{0}(\lambda t)
$$

The differential of a simple Poisson counting process satisfies:

$$
d P_{t}=\lambda d t
$$

with $\lambda>0$ and initial conditions $P\left(0^{+}\right)=0$ with probability one. The simplest approach to view the Poisson processes is to consider these differential stochastic processes as increments, i.e.:

$$
d P_{t}=P(t+d t)-P(t)
$$

for infinitesimal increments in time $d t$. We verify that the Poisson process $P_{t}$ is quite different from continuous diffusion processes, primarily because of its discontinuity property, and the property that multiple jumps are highly unlikely during small increments of time $d t$.

The sample path of a Poisson process with $\lambda=2$ is displayed in Figure 8.

\subsubsection{Bernoulli process}

The Bernoulli process is the discrete time counterpart of the Poisson process. It consists of finite or infinite sequence of independent random variables $X_{t}, t=1, \ldots, T$ such that:

$$
X_{t}=\left\{\begin{aligned}
1 & \text { with } \text { prob }=p \\
-1 & \text { with } \text { prob }=1-p
\end{aligned}\right.
$$

In practice, this model corresponds to a regular sampling period $S$ for which observations are missing at random, with failure probability $1-p$. The regular sampling period corresponds to $p=1$. Random variables associated with the Bernoulli process include:

- The number of successes in the first $n$ trials: this has a binomial distribution.

\footnotetext{
${ }^{29}$ There are other qualitative features that characterize real market log-return distributions that cannot be reproduced by the pure-diffusion model but can be modeled, in part, by adding jumps to the diffusion process. First, real markets have negatively skewed log-return distributions: they are found to be pessimistic due to more negative logreturns (including crashes) than positive log-returns. Second, real markets distributions are found to be leptokurtic: the distribution is more peaked at the maximum, and consequently has fatter tails than the normal distribution. The third characteristic is the volatility smile, which refers to the curvature of the implied volatility (e.g. volatility implied by the log-normal Black-Scholes formula) versus the strike price.
} 


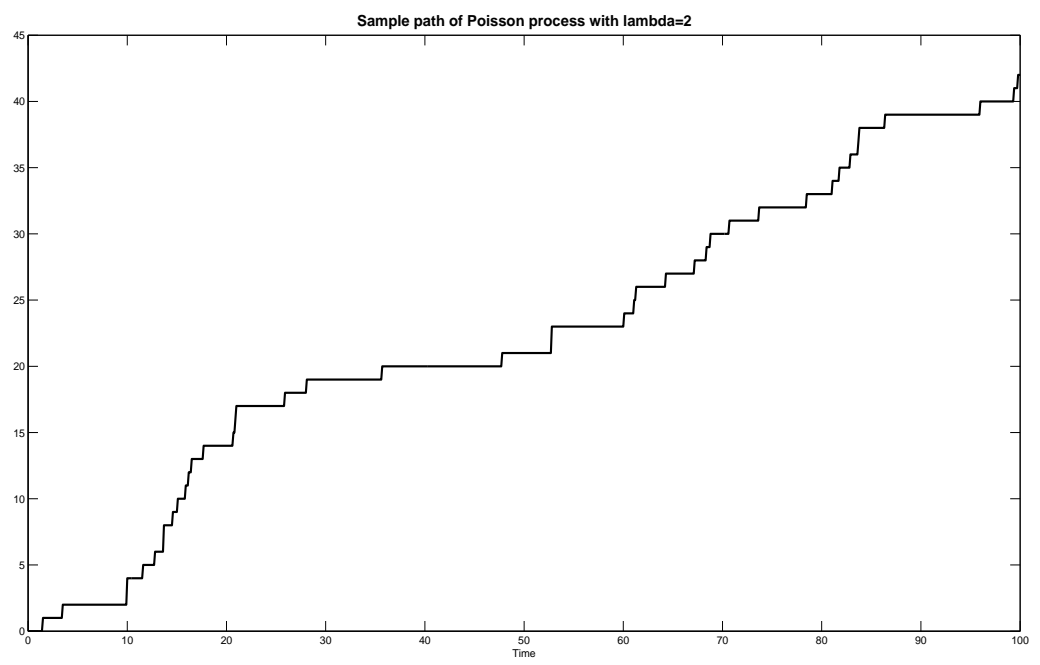

Figure 8: Numerical illustration of Poisson process

- The number of trials needed to get $r$ successes: this has a negative binomial distribution.

- The number of trials needed to get one success: this has a geometric distribution, which is a special case of the negative binomial distribution.

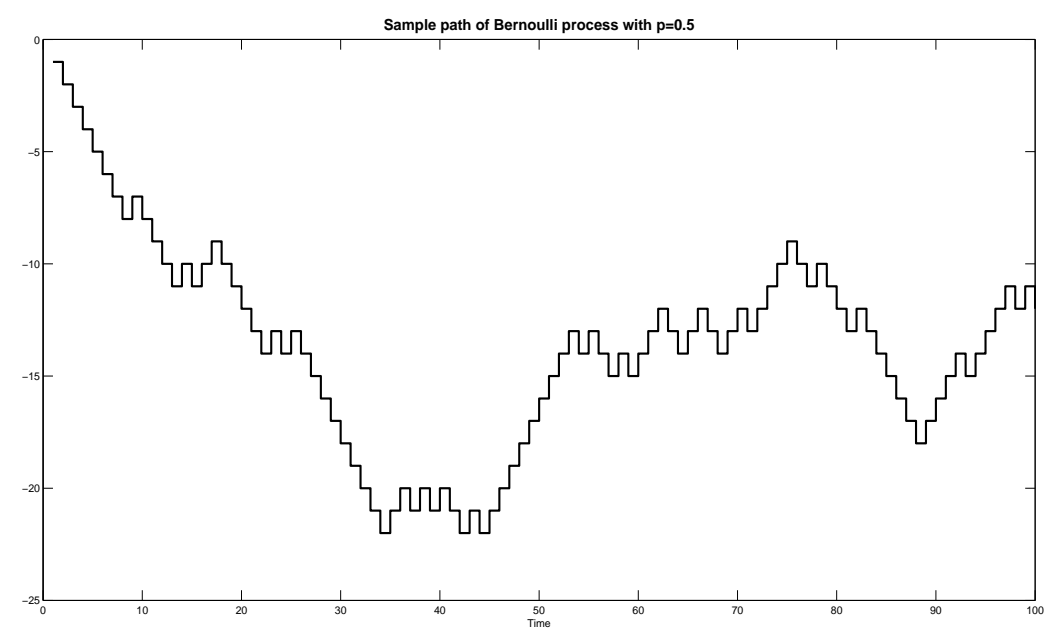

Figure 9: Numerical illustration of Bernoulli process

As an example, the numerical simulation of a Bernoulli process with $p=0.5$ from a discrete uniform $X_{t} \sim \mathcal{U}_{d}(-1,1)$ is given in Figure 9. 


\subsubsection{Lévy process}

Lévy processes can be thought of as a combination of a diffusion process and a jump process. Both Brownian motion (i.e. a pure diffusion process) and Poisson processes (i.e. pure jump processes) are Lévy processes. As such, Lévy processes represent a tractable extension of Brownian motion to infinitely divisible distributions. In addition, Lévy processes allow the modeling of discontinuous sample paths, whose properties match those of empirical phenomena such as financial time series.

There have been many efforts to apply Lévy processes, such as the CGMY model (Carr et al. (2003)), the variance gamma (VG) model (Carr and Madan (1999)), and the Normal Inverse Gaussian (NIG) model (Rydberg (1997)).

A $d$-dimensional Lévy process is a stochastic process $\left\{X_{t}, t \geq 0\right\}$ taking values in $\mathbb{R}^{d}$ with the following properties:

1. Independent increments: For any $t_{1}<t_{2} \leq t_{3}<t_{4}$, the random variables $X_{t_{4}}-X_{t_{3}}$ and $X_{t_{2}}-X_{t_{1}}$ are independent.

2. Stationarity: The law of $X_{t+h}-X_{t}$ does not depend on $t$.

3. Stochastic continuity: when the process coefficients are not constant, then the process will in general not be stationary, as the preceding condition requires. For many real problems, such as in financial markets, the time-dependence of process coefficients is important (Hanson and Westman (2002)).

4. Zero initial value: $X_{0}=0$ almost surely.

A Lévy process can be seen as a continuous time generalization of a random walk process. Indeed, the process observed at time $0=t_{0}<t_{1}<t_{2}<\ldots$ forms a random walk:

$$
X_{t_{n}}=\sum_{i=1}^{n}\left(X_{t_{i}}-X_{t_{i-1}}\right)
$$

whose increments $\left\{X_{t_{i}}-X_{t_{i-1}}\right\}$ are independent. Let $N([0, t] \times A)$ denote the number of jumps of $X$ during the interval $[0, t]$ whose size lies in the ensemble $A$, excluding 0 . Let $\Delta X_{t}$ denote the size of the jump of the process at time $t$. The measure $\nu$ defined by:

$$
\nu(A)=N([0,1] \times A), \quad\left\{t \in[0,1]: \Delta X_{t} \neq 0, \Delta X_{t} \in A\right\}
$$

is called the Lévy measure of $X_{t}$. The random measure $N(d t, d x)$ is called the jump measure. We observe that Lévy processes are essentially jump-diffusion processes, but are extended to processes with infinite jump-rates.

A numerical simulation of a Lévy process with $\nu=0.01$ can be observed in Figure 10. 
Sample path of Lévy process with nu=0.01

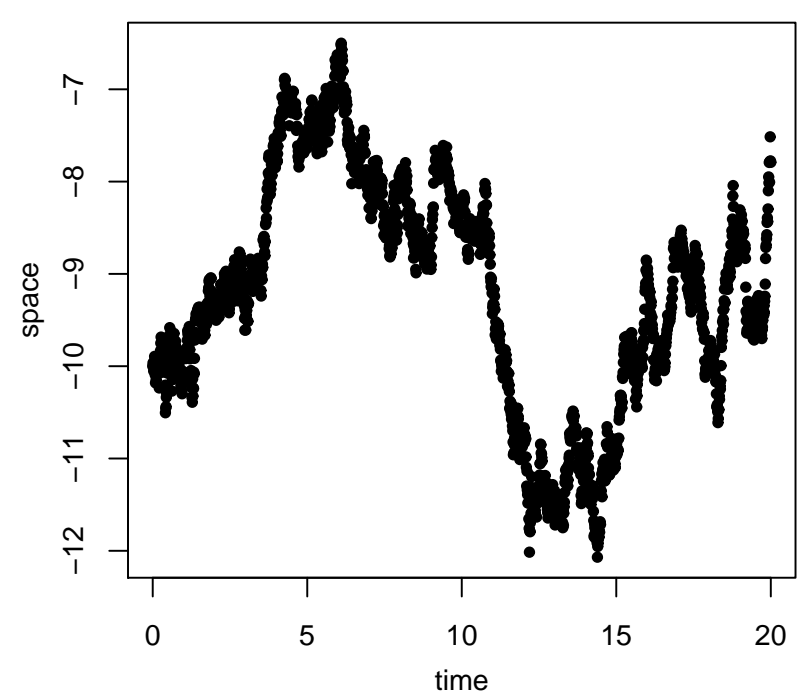

Figure 10: Numerical illustration of Lévy process 